\title{
Estudo Experimental e Teórico dos Processos no Plasma ICP-AES Não-Estacionário
}

R. TOUkhVatoulline ${ }^{1}$ H. BOnadiman, M. PiEniZ, Departamento de Física, Estatísitica e Matemática, Universidade de Ijuí -UNIJUÍ, DeFEM, Rua São Francisco, 501, Cx.P. 560, 98700-000 Ijuí, RS, Brasil.

\begin{abstract}
Resumo. Foram experimentalmente estudadas as pulsações transversais da tocha de plasma com simetria cilíndrica ICP-AES (Iductively Coupled Plasma Atomic Emission Spectroscopy). Os resultados mostraram que no plasma ICP-AES as pulsações transversais estão praticamente ausentes, mas existem pulsações de intensidade de radiação em razão da grande freqüência da corrente elétrica. Assim, esses resultados permitiram propor, para a tocha de plasma ICP-AES, um modelo matemático que prevê apenas a presença de oscilações da intensidade de radiação. Neste modelo supõe-se a tocha de plasma como sendo estacionária no espaço, mas com pulsações de temperatura em cada ponto da tocha. Com este modelo e a partir das intensidades laterais (observadas pelo espectrômetro) foram calculadas as distribuições radiais da intensidade de radiação e da temperatura. Os cálculos foram feitos para o modelo estacionário e não-estacionário supondo que as pulsações de temperatura sigam a distribuição de Gauss. Para o estudo foi escolhida a raia espectral ArI 435,8 nm. O efeito das pulsações foi estudado para diferentes valores de desvio-padrão da temperatura. Os resultados mostram que as pulsações, dentro do plasma, podem interferir significativamente nos resultados experimentais e ser esta a causa da diferença entre os cálculos teóricos e as medidas experimentais.
\end{abstract}

\section{Introdução}

Um dos mais importantes parâmetros de plasma é a temperatura. Conhecendose a temperatura e sua distribuição espacial pode-se determinar as características do plasma, tais como os coeficientes de condutividade, de calor, de viscosidade, as concentrações de elétrons, de íons e de átomos, as velocidades de reação dos diferentes processos físico-químicos, as propriedades termodinâmicas, entre outras. Os cálculos teóricos e as determinações experimentais da temperatura são realizados supondo que o plasma é estacionário no tempo e no espaço. No entanto, observa-se diferenças entre os cálculos teóricos da distribuição radial da temperatura e as medidas experimentais $[1,6]$. Para estas diferenças existem explicações, como desvio do equilíbrio térmico local, etc. Outra causa para as diferenças entre os resultados teóricos e experimentais, que ainda não foi suficientemente estudada, é a instabilidade do plasma. Numerosas medidas das pulsações de arcos elétricos em geradores

\footnotetext{
${ }^{1}$ rafkat@main.unijui.tche.br, fax: 0553329100
} 
de plasma mostram que o plasma não é estacionário. Ele apresenta pulsações de tocha, de corrente, de tensão e de intensidade de radiação [7]. Por Dautov et al. [7] foram estudadas as pulsações espaciais do arco elétrico estabilizado e as pulsações da intensidade da radiação no plasmotron. O esquema das pesquisas de oscilações da intensidade de radiação do plasma em plasmotron com arco elétrico estabilizado, é apresentado na Figura 1. A radiação do plasma (1) é projetada, com o auxílio de uma lente (2), na fenda de entrada do espectrógrafo (3). Na saída do espectrógrafo está o foto-sensor FEP-1 (4) que transforma o sinal ótico em sinal elétrico e, na tela do osciloscópio (5), são observadas as oscilações da intensidade do plasma. As oscilações transversais da tocha foram estudadas com um foto-registrador de alta velocidade $(25 \mathrm{~m} / \mathrm{s})$. As posições do arco elétrico foram fotografadas com o auxílio de uma lente (6) no filme do foto-registrador (7).

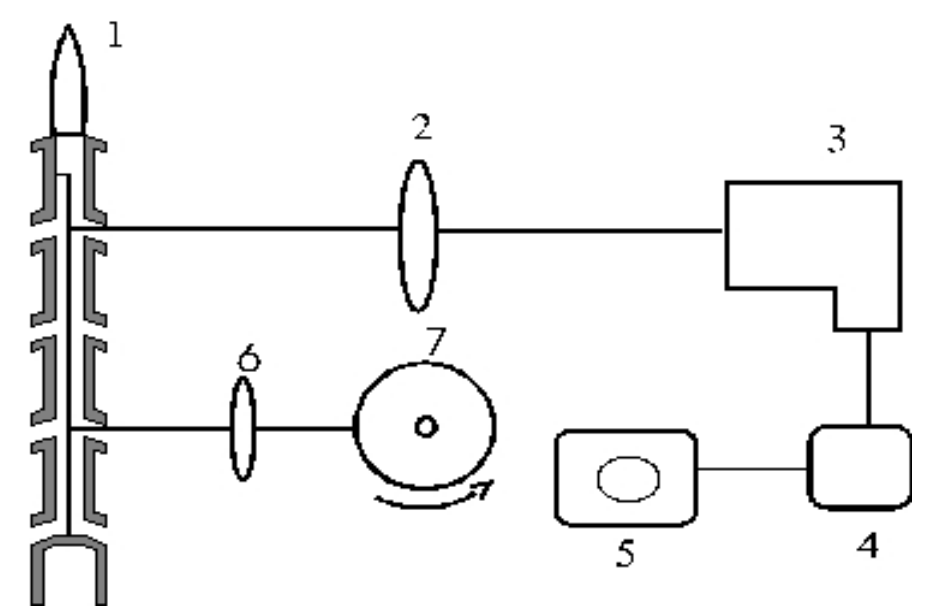

Figura 1: 1- plasmotron, 2 e 6 - lentes, 3- espectrógrafo, 4 - foto-sensor FEP-1, 5 osciloscópio, 7 - foto-registrador.

Na Figura 2 são apresentados o oscilograma das pulsações do plasma para a raia espectral NI 493,5 nm (Fig.2a) e o registrograma das pulsações transversais da tocha de plasma (Fig.2b). Pode-se ver, por esta figura, que dentro do plasma existem pulsações da intensidade da raia espectral e da tocha. O tratamento estatístico destas pulsações mostrou que elas seguem uma distribuição de Gauss.

Resultados experimentais mostraram que dentro do plasma existem dois tipos de pulsações: 1. pulsações de intensidade de radiação e 2. pulsações espaciais da tocha. A amplitude destas pulsações varia entre $10 \%$ e $50 \%$.

A tocha de plasma ICP-AES, objeto de nosso estudo, também é não-estacionária. A instabilidade espacial do plasma desta tocha é menor do que no plasma com arco estabilizado. Ela tem instabilidade hidrogazodinâmica causada pela interação da tocha do plasma com o fluxo de Argônio frio e, ainda, pela instabilidade temporária em cada ponto do plasma em razão da freqüência ser da ordem de $10 \mathrm{Mhz}$. Es- 


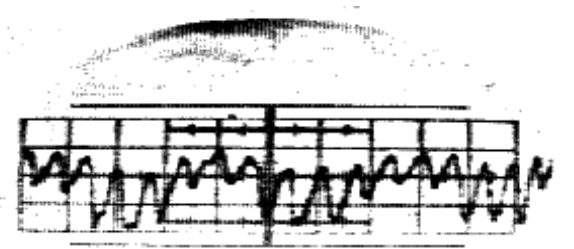

a

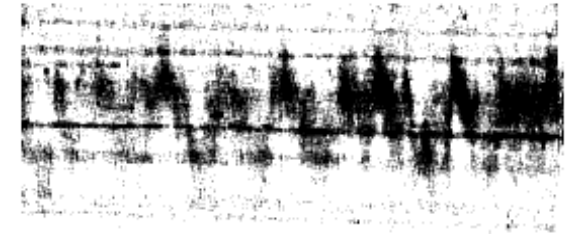

b

Figura 2: Oscilograma das oscilações da intensidade da raia espectral NI 493,5 nm (a) e registrograma das pulsações transversais da tocha do plasma com a simetria cilíndrica (b)

tas pulsações influem nos resultados da determinação de intensidades laterais e, como conseqüência, na distribuição radial da temperatura pelos métodos espectroscópicos. O grau de instabilidade depende de diferentes fatores: dos parâmetros elétricos, da turbulência do plasma e da forma da fonte de plasma. Os resultados de pesquisas das pulsações em geradores de plasma mostram que as causas principais que influem na precisão da determinação da temperatura radial são as pulsações transversais da tocha de plasma e as pulsações da temperatura dentro do plasma. O modelo da tocha de plasma em presença de pulsações espaciais foi estudado em [8], onde foi obtido a solução exata da equação de Fredholm para um caso particular. Os resultados deste trabalho mostraram que pulsações espaciais podem influir significativamente nos resultados da determinação da temperatura pelos métodos espectrométricos.

No trabalho que apresentamos foram estudados experimentalmente as pulsações espaciais no plasma ICP-AES. Teoricamente foi considerado um outro caso limite de modelo: o plasma com simetria cilíndrica como sendo estacionário no espaço mas com pulsações temporais de temperatura em cada ponto dentro da tocha. Para este modelo foram calculados, a partir das intensidades laterais de radiação, as distribuições radiais da temperatura para o plasma estacionário e não-estacionário. O efeito das pulsações foi estudado para diferentes valores de desvio-padrão da temperatura. Os cálculos foram realizados para a raia espectral ArI 435,8 nm e as temperaturas no eixo da tocha $8000 \mathrm{~K}, 12000 \mathrm{~K}$ e $16000 \mathrm{~K}$.

\section{Parte Experimental}

Os experimentos para o estudo das pulsações espaciais da tocha de plasma foram realizados num espectrômetro de emissão atômica com plasma indutivamente acoplado (ICP-AES), fabricado pelo Spectro Analitical Instruments (Alemanha), com as características que a seguir especificaremos. Potência: $1,2 \mathrm{KW}$; freqüência: $27,12 \mathrm{MHz}$; fluxo de gás de refrigeração: 12 l/min; gás auxiliar (argônio): 0,8 l/min; gás neb- 
ulizador: 1,0 l/min; aspiração da amostra: $2 \mathrm{ml} / \mathrm{min}$; altura da fibra ótica: $1 \mathrm{~mm}$ acima da bobina; tempo de integração: $10 \mathrm{~s}$. Na investigação foram utilizados dois equipamentos auxiliares construídos para esta finalidade: um foto-registrador e um aparelho ótico rotativo. O foto-registrador é constituído de uma cuba cilíndrica de PVC com $20 \mathrm{~cm}$ de diâmetro e $9 \mathrm{~cm}$ de altura. Esta cuba possui uma fenda de aproximadamente $20 \times 0,5 \mathrm{~mm}$ pela qual passa as radiações provenientes do plasma e que incidem sobre um filme muito sensível colocado ao longo das paredes internas de outra cuba cilíndrica de plástico transparente, de $15 \mathrm{~cm}$ de diâmetro e $5 \mathrm{~cm}$ de altura, presa no interior da primeira. O conjunto foi acoplado sobre um pequeno motor de $3500 \mathrm{RPM}, 220 \mathrm{~V}, 60 \mathrm{~Hz}$ que, ao ser ligado, possibilita que o filme gire numa velocidade aproximada de $27 \mathrm{~m} / \mathrm{s}$ e seja capaz de registrar processos que acontecem muito rapidamente $\left(10^{-5} s\right)$, como as oscilações de uma tocha de plasma. Em frente à fenda do foto-registrador foi adaptado um diafragma de máquina fotográfica que regula o tempo de exposição do filme às radiações da tocha de plasma que pode ser variado de $1 / 30$ a 1/500 s. Em frente ao foto-registrador foi posicionado o aparelho ótico rotativo constituído de uma caixa de madeira, móvel para girar, de $11 \times 13 \times 21 \mathrm{~cm}$ e presa a um suporte também de madeira. Entre o foto-registrador e o aparelho ótico rotativo foi colocada uma lente convergente com a função de focalizar, sobre a película registradora, a imagem da tocha de plasma para a obtenção de uma fotografia mais nítida e mais detalhada, o que irá facilitar a interpretação e a análise dos resultados. No aparelho ótico rotativo foram colados três espelhos planos, um na parte superior da caixa e, os outros dois, dispostos na entrada e na saída da mesma formando, entre si, um ângulo de $120^{\circ}$, conforme mostra o esquema da figura 3. Sua função é girar a imagem da tocha de plasma para ser captada pelo foto-registrador no ângulo desejado.

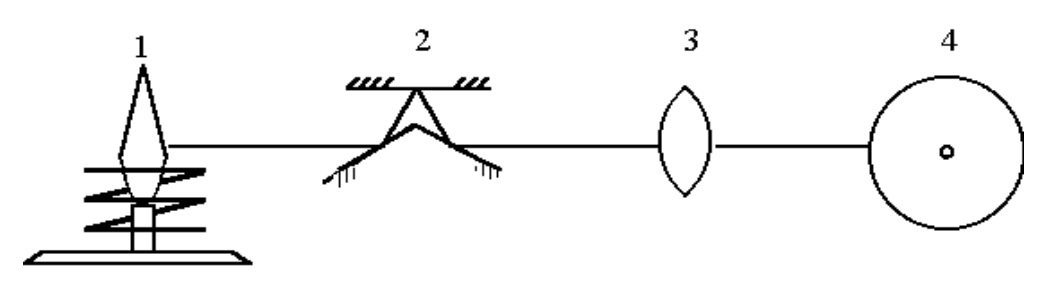

Figura 3: Esquema do experimento: 1 - tocha de plasma de ICP-AES, 2 - aparelho ótico rotativo, 3 - lente convergente, 4 - foto-registrador.

Para o experimento em questão, a caixa do aparelho ótico rotativo é girada de $45^{0}$ permitindo que a imagem da tocha de plasma gire num ângulo de $90^{\circ}$ e seja registrada no filme do foto-registrador numa posição horizontal o que possibilita a visualização das pulsações espaciais, se houverem, e de intensidade luminosa. A Figura 4 mostra um registrograma da imagem da tocha de plasma do ICP-AES 
obtida, num dos experimentos realizados, no filme do foto-registrador.

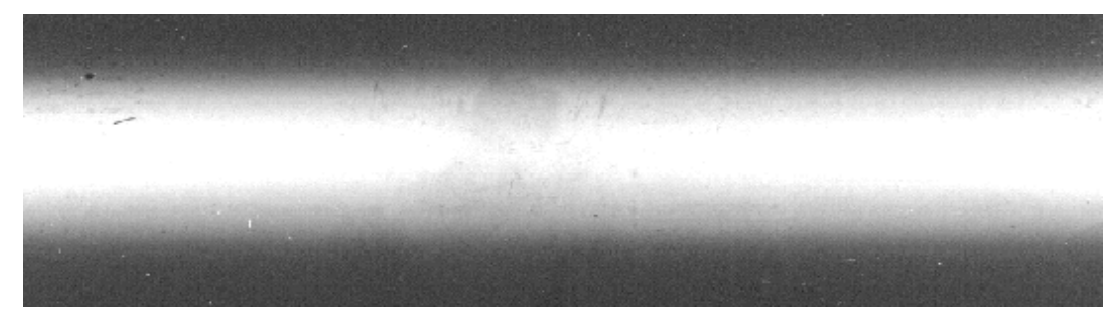

Figura 4: Registrograma da tocha de plasma do ICP-AES num dos experimentos realizados.

No registrograma mostrado na Figura 4, se comparado com o registrograma mostrado na Figura 2b, percebe-se a ausência quase total de pulsações transversais espaciais. Sabe-se, no entanto, em função da frequência de $27 \mathrm{MHz}$, que no plasma ICP-AES existem pulsações temporais, semelhantes às mostradas na Figura 2a.

\section{Plasma Estacionário}

A temperatura da tocha do plasma oticamente fino em equilíbrio térmico local (ETL) com a simetria cilíndrica pode ser determinada pelo método da intensidade absoluta da raia espectral e com o auxílio das relações:

$$
\begin{gathered}
\varepsilon_{\nu}=\frac{1}{4 \pi} A_{n}^{m} \frac{g_{m}}{Z} n_{a} e^{-\frac{E m}{k T}} h v, \\
I_{\nu}(x)=2 \int_{x}^{R} \frac{\varepsilon_{\nu}(r) r d r}{\sqrt{r^{2}-x^{2}}}, \quad 0 \leq r \leq R,
\end{gathered}
$$

onde $\varepsilon_{\nu}$ é a intensidade local da raia, $I_{\nu}(x)$ é a intensidade lateral, $A_{n}^{m}$ é a probabilidade de transição do nível $m$ para o nível $n, g_{m}$ é o fator de degerescência do nível $m, Z$ é a função partição, $n_{a}$ é a concentração de átomos no plasma, $E_{m}$ é a energia do nível $m, k$ é a constante de Boltzmann, $T$ é a temperatura, $h$ é a constante de Planck, $\nu$ é a frequência e $R$ é o raio da tocha. $I_{\nu}(x)$ é determinada experimentalmente e $\varepsilon_{\nu}(r)$ é determinada através da integral de Abel

$$
\varepsilon_{\nu}(r)=-\frac{1}{\pi} \int_{r}^{R} \frac{d I_{\nu}(x)}{d x} \frac{d x}{\sqrt{x^{2}-r^{2}}} .
$$

As integrais nas equações (3.3) ou (3.2) podem ser resolvidas por métodos numéricos. Colocando os valores das intensidades radiais $\varepsilon_{\nu}(r)$ na fórmula (3.1), determina-se a distribuição radial da temperatura do plasma. Na mesma equação 
(3.1) $n_{a}$ e $Z$ dependem da temperatura. Para o plasma onde existem somente átomos de Argônio uma vez ionizados, $n_{a}$ é dada pela equação

$$
n_{a}(T)=\frac{p}{k T}+2 S(T)-2 \sqrt{S^{2}(T)-S(T) \frac{p}{k T}},
$$

onde

$$
S(T)=4,83.10^{23} \frac{Z_{a}}{Z_{+}} e^{-\frac{\chi}{k T}} .
$$

Aqui $S(T)$ é a constante de ionização, $p$ é a pressão, $k$ é a constante de Boltzmann, $\chi$ é a energia da ionização, e $Z_{a}$ e $Z_{+}$são as funções de partição para íons e átomos, respectivamente. Para o cálculo das funções de partição $Z(T)$ utilizamos a relação

$$
Z=\sum_{i=1}^{\infty} g_{i} e^{-\frac{E_{i}}{k T}}
$$

Os valores do fator de degerescência e a energia de excitação para os cálculos foram retirados de [9]. Os cálculos foram feitos para pressão $\mathrm{p}=10^{5} \mathrm{~Pa}$, no intervalo de temperaturas $8000 \mathrm{~K}-26000 \mathrm{~K}$.

Para os cálculos da intensidade local da raia espectral em função da temperatura foi escolhida a raia espectral Ar I com o comprimento de onda $\lambda=415,8 \mathrm{~mm}$ que frequentemente é utilizada na determinação da temperatura. Os resultados dos cálculos estão apresentados na Figura 5 (curva 1).

\section{Plasma Não-Estacionário. Pulsações da Tem- peratura}

Dada a tocha de plasma com simetria cilíndrica, conhecida sua distribuição lateral da intensidade e, ainda, admitindo que cada ponto da tocha tem flutuações de temperatura que distribuem-se de acordo com a densidade de probabilidade de Gauss, temos

$$
f\left(T-T_{m}\right)=\frac{1}{\sigma \sqrt{2 \pi}} e^{-\frac{\left(T-T_{m}\right)^{2}}{2 \sigma^{2}}},
$$

onde $\sigma$ é o desvio padrão da temperatura e $T_{m}$ é a temperatura média. Vamos calcular como influem estas pulsações na intensidade local $\varepsilon_{\nu}(T)$. Com a consideração de (4.1) $\bar{\varepsilon}$ pode ser determinada como sendo

$$
\bar{\varepsilon}_{\nu}\left(T_{m}\right)=\int_{-\infty}^{\infty} \varepsilon_{\nu}(T) f\left(T-T_{m}\right) d T .
$$

A integração de (4.2) pode ser feita em pequeno intervalo de temperaturas, pois $f\left(T-T_{m}\right)$ diminui rapidamente com o aumento de $\left(T-T_{m}\right)$. Na Figura 5 são apresentados os resultados de cálculos de $\bar{\varepsilon}_{\nu}\left(T_{m}\right)$ para $\sigma=1000 \mathrm{~K}(2)$ e $\sigma=3000$ $\mathrm{K}(3)$. 


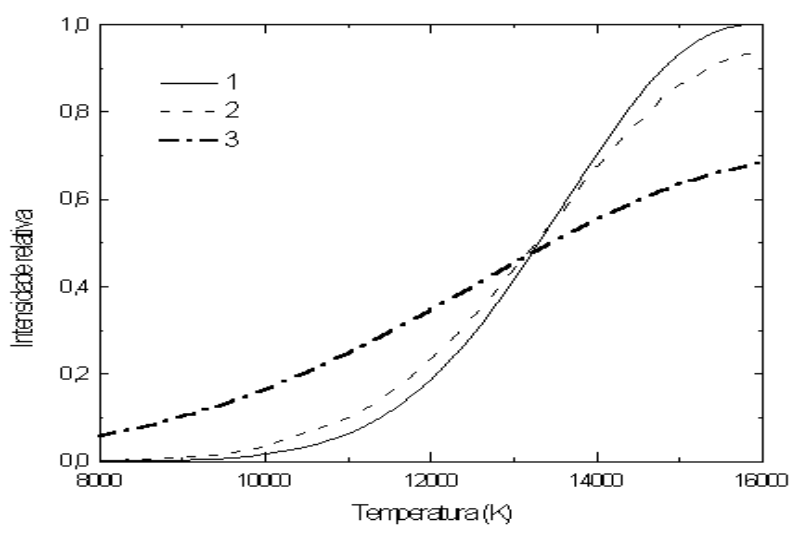

Figura 5: Intensidade relativa da raia $A r I 415,8 \mathrm{~nm}$ em função da temperatura na ausência (1) e na presença de pulsações $(2) \sigma=1000 \mathrm{~K} \mathrm{e} \mathrm{(3)} \sigma=3000 \mathrm{~K}$.

A curva (1), neste gráfico, corresponde à relação $\varepsilon_{\nu}(T)$ (eq.(3.1)) na ausência de pulsações. Nele pode-se ver que os valores reais $\varepsilon$ diferem dos valores observados $\bar{\varepsilon}$. Esta diferença depende das intensidades das pulsações $(\sigma)$ e do intervalo de temperatura. Para raia de Argônio ArI435, $8 \mathrm{~nm}$ a diferença entre $\bar{\varepsilon}_{\nu}\left(T_{m}\right)$ e $\varepsilon_{\nu}(T)$ é maior nas regiões de temperaturas de $8000 \mathrm{~K}$ e $16000 \mathrm{~K}$ e $\sigma=3000 \mathrm{~K}$.

\section{O Cálculo da Temperatura Radial}

Para determinar a distribuição radial da temperatura escolhemos a distribuição lateral da intensidade $I_{\nu}(x)$, observada pelo espectrômetro, na seguinte forma:

$$
I_{\nu}(x)=I_{\nu}(0) e^{-\alpha x^{2}},
$$

onde $I(0)$ é a intensidade no eixo da tocha, $\alpha$ é um coeficiente. Colocando (5.1) na equação (3.3) obtemos a intensidade radial $\varepsilon_{\nu}(r)$ e, utilizando os resultados do cálculo de $\varepsilon_{\nu}(T)$ (Fig.5), foram determinados os valores relativos de $\varepsilon_{\nu}^{\prime}(r)$ referentes à ausência e à presença de pulsações no plasma. Os valores de $\varepsilon_{\nu}^{\prime}(r)$ foram calculados para a temperatura no eixo da tocha de $8000 \mathrm{~K}, 12000 \mathrm{~K}$ e $16000 \mathrm{~K}$. Os resultados dos cálculos para a temperatura no eixo de $12000 \mathrm{~K}$ são apresentados na Tabela 1. 
Tabela 1:

\begin{tabular}{|c|c|c|c|c|c|}
\hline $\mathrm{r} / \mathrm{R}$ & $I_{\nu}(x)$ & $\varepsilon_{\nu}(r)$ & $\varepsilon_{\nu}^{\prime}(r)$ & $\varepsilon_{\nu}^{\prime}(r), \sigma=1000 \mathrm{~K}$ & $\varepsilon_{\nu}^{\prime}(r), \sigma=3000 \mathrm{~K}$ \\
\hline 0 & 1 & 1,784 & 0,187 & 0,233 & 0,346 \\
\hline 0,05 & 0,975 & 1,740 & 0,182 & 0,229 & 0,343 \\
\hline 0,1 & 0,905 & 1,614 & 0,169 & 0,215 & 0,335 \\
\hline 0,15 & 0,798 & 1,425 & 0,149 & 0,195 & 0,322 \\
\hline 0,2 & 0,670 & 1,196 & 0,125 & 0,171 & 0,305 \\
\hline 0,25 & 0,535 & 0,955 & 0,100 & 0,143 & 0,284 \\
\hline 0,3 & 0,406 & 0,725 & 0,076 & 0,114 & 0,261 \\
\hline 0,35 & 0,294 & 0,524 & 0,055 & 0,091 & 0,235 \\
\hline 0,4 & 0,202 & 0,360 & 0,038 & 0,072 & 0,211 \\
\hline 0,45 & 0,132 & 0,235 & 0,025 & 0,053 & 0,186 \\
\hline 0,5 & 0,082 & 0,146 & 0,0153 & 0,033 & 0,162 \\
\hline 0,55 & 0,048 & 0,086 & 0,009 & 0,022 & 0,139 \\
\hline 0,6 & 0,027 & 0,049 & 0,005 & 0,013 & 0,119 \\
\hline 0,65 & 0,015 & 0,026 & 0,003 & 0,009 & 0,100 \\
\hline 0,7 & 0,007 & 0,013 & $1,40 \mathrm{E}-03$ & 0,005 & 0,084 \\
\hline 0,75 & $3,61 \mathrm{E}-03$ & $6,41 \mathrm{E}-03$ & $6,71 \mathrm{E}-04$ & $3,29 \mathrm{E}-03$ & $6,97 \mathrm{E}-02$ \\
\hline 0,8 & $1,66 \mathrm{E}-03$ & $2,93 \mathrm{E}-03$ & $3,07 \mathrm{E}-04$ & $1,81 \mathrm{E}-03$ & $5,99 \mathrm{E}-02$ \\
\hline 0,85 & $7,28 \mathrm{E}-04$ & $1,28 \mathrm{E}-03$ & $1,34 \mathrm{E}-04$ & $1,11 \mathrm{E}-03$ & $5,53 \mathrm{E}-02$ \\
\hline 0,9 & $3,04 \mathrm{E}-04$ & $5,14 \mathrm{E}-04$ & $5,38 \mathrm{E}-05$ & $7,85 \mathrm{E}-04$ & $5,31 \mathrm{E}-02$ \\
\hline 0,95 & $1,20 \mathrm{E}-04$ & $1,80 \mathrm{E}-04$ & $1,89 \mathrm{E}-05$ & $6,43 \mathrm{E}-04$ & $5,22 \mathrm{E}-02$ \\
\hline
\end{tabular}

Nesta tabela, $\varepsilon_{\nu}^{\prime}(r)$ é igual a $\varepsilon_{\nu}(r) / \mathrm{k}$, onde $\mathrm{k}=\varepsilon_{\nu}(r) / \varepsilon_{\nu}(T)$ para o eixo do plasma $(r=0$ e $T=12000 \mathrm{~K})$.

As distribuições radiais da temperatura foram obtidas comparando os valores das colunas 4, 5 e 6 da tabela com a curva 1 da Fig.5. Os cálculos foram feitos para a temperatura no eixo da tocha de $8000 \mathrm{~K}, 12000 \mathrm{~K}$ e $15000 \mathrm{~K}, I(0)=1,0$ e $\alpha=5,0$ e $10,0, \sigma=1000 \mathrm{~K}$ e $3000 \mathrm{~K}$. Os resultados, para $\alpha=10$, 0 , são apresentados na Figura $6(6 \mathrm{a}, 6 \mathrm{~b}, 6 \mathrm{c})$.

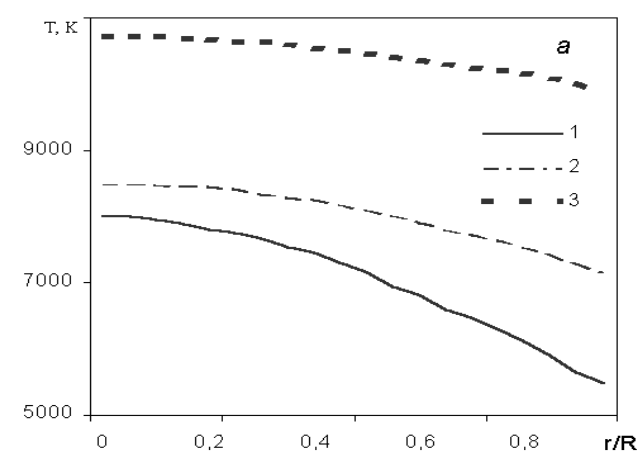



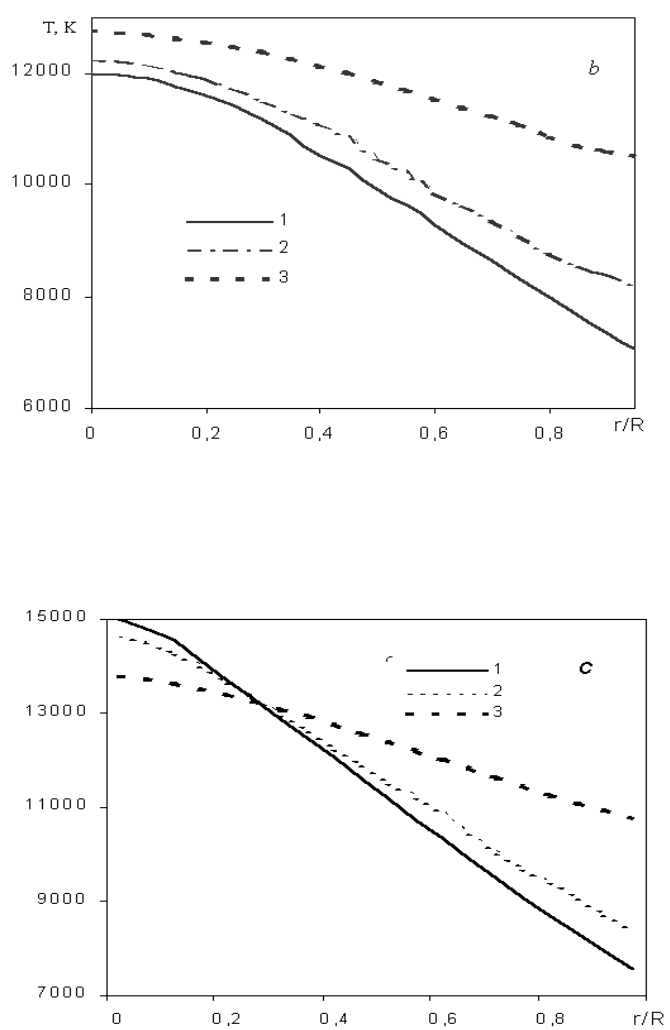

Figura 6: Distribuição radial de temperatura em ausência de pulsações (1) e em presença delas $(2,3)$ para $\sigma=1000$ (2) e 3000 (3).

Nesta figura, a curva 1 corresponde à temperatura radial real da tocha do plasma. As curvas (2) e (3) são distribuições da temperatura quando $\sigma=1000 \mathrm{~K}$ e $3000 \mathrm{~K}$, respectivamente.

\section{Análise dos Resultados e Conclusões}

Os resultados obtidos experimentalmente mostraram que na tocha de plasma ICPAES as pulsações espaciais estão praticamente ausentes. O plasma é bastante estável no espaço mas existem pulsações de intensidade da radiação em razão de sua alta freqüência, de 10 a $30 \mathrm{MHz}$.

Os resultados experimentais mostraram, também, que a tocha de plasma ICPAES pode ser considerada estacionária no espaço tendo, apenas, pulsações de temperatura. Para este modelo de plasma foram calculadas as distribuições radiais da 
temperatura supondo que as pulsações sigam a distribuição de Gauss.

Na Fig. 6 deste trabalho pode-se observar que as pulsações de temperatura podem influir significativamente nos resultados experimentais das medidas das temperaturas radiais. Observa-se que as curvas de temperatura em presença de pulsações (curvas pontilhadas) diminuem mais lentamente que a curva estabelecida com a ausência de pulsações (curva cheia). Para as temperaturas no eixo da tocha de 8000 $\mathrm{K}$ e $12000 \mathrm{~K}$ (6a e 6b) as curvas de temperaturas radiais são mais altas em todo o intervalo de temperaturas do que as curvas reais. A diferença entre os resultados observados e verdadeiros dependem do desvio-padrão $\sigma$. Com o aumento do $\sigma$ aumenta também esta diferença. O erro na determinação da temperatura radial pelos métodos espectrométricos pode atingir $4000 \mathrm{~K}$ ( Fig. 6a, $\sigma=3000 \mathrm{~K}$ ). Para a temperatura no eixo de tocha de $15000 \mathrm{~K}$ as curvas, em presença de pulsações, diminuem mais lentamente mas, na região acima de $13200 \mathrm{~K}$, é inferior que as temperaturas reais e somente abaixo desta temperatura elas são superiores.

Comparando as curvas nas Figuras 6 e 5 pode-se ver que a forma das distribuições radiais observadas depende da temperatura no eixo da tocha. No intervalo de temperaturas menores que $13200 \mathrm{~K}$ as distribuições radiais observadas são superiores que as reais e, para maiores que $13200 \mathrm{~K}$, são menores.

Portanto, as pulsações dentro do plasma podem interferir nos resultados experimentais e ser a causa da diferença entre os valores obtidos pelos cálculos teóricos e os valores obtidos experimentalmente.

\section{Referências}

[1] A. Montaser and D.W. Golightly, "Inductively Coupled Plasmas in Analytical Atomic. Spectrometry", 2nd Edition, VCH Publishers, New York Weinheim Cambridge, 1992.

[2] S.V. Dresvin, "Physics and Technology of Low-Temperature Plasmas", (translation from Russian), Iowa State University Press, 1977.

[3] J. Mostaghimi and M.I. Boulos, J. Appl. Phys., 68 (1990), 2643-2648.

[4] J. Mostaghimi, P. Proulx and M.I. Boulos, J. Appl. Phys., 61 (1987), 1753-1760.

[5] R.C. Miller and J.A. Richard, J. Appl. Phys., 40 (1969), 5260-5273.

[6] D.C. Pridmore-Brown, J. Appl. Phys., 41 (1970), 3621-3625.

[7] G. Dautov, R. Toukhvatoulline and N. Zalialov, in "Procedures of the XX International Coloquium on Atomic and Molecular Spectroscopy", Prague, 1977.

[8] R. Toukhvatoulline, G. Feldmann, Effect of pulsations of a plasma torch on the radial distribution of temperature Fresenius, J. Anal. Chem., (1999).

[9] A.R. Striganov and M.S Sventitskii, "Tables of Spectral Lines of Neutral and Ionized Atoms", IFI / Plenum, New York, 1968. 